\title{
Influence of silver and gold nano particles on luminance properties of n-hexyloxy-cynobiphenyl liquid crystal through image analysis
}

\author{
D. R. Roopa ${ }^{1}$, C. M. Subhan ${ }^{2}$, K. Fakruddin ${ }^{3, *}$, P. Pardhasaradhi ${ }^{4}$, B. T. P. $\operatorname{Madhav}^{4}$, G. Srilekha ${ }^{4}$ \\ ${ }^{1}$ Department of Physics, SJB Institute of Technology, Bengaluru, Karnataka, India \\ ${ }^{2}$ Department of Physics, SV Degree and PG College, Anantapur, Andhra Pradesh, India \\ ${ }^{3}$ Department of Physics, Ghousia College of Engineering, Ramanagaram, Karnataka, India \\ ${ }^{4}$ Department of ECE, Koneru Lakshmaiah Education Foundation, Vaddeswaram, Guntur, India \\ *drfakruddin2008@gmail.com
}

PACS 42.70.Df, 42.79.Kr, 87.15.mq.

DOI 10.17586/2220-8054-2021-12-4-453-461

\begin{abstract}
In this work, gold and silver nanoparticles are separately dispersed in n-hexyloxy-cyanobiphenyl (6O.CB) liquid crystal in the ratio 1:100. The optical textures of pure and nano-dispersed compounds are recorded by using polarizing optical microscope. Differential scanning calorimeter (DSC) is used to compute enthalpy and transition temperature values. Due to the dispersion of nanoparticles, the clearing temperature of the compounds are slightly reduced but there is no significant change in the nematic phase exhibited by pure 6O.CB. Further characterization is done by various spectroscopic techniques like scanning electron microscopy (SEM) and X-ray diffraction (XRD). The optical textures have been analyzed using MATLAB software for the evaluation of display parameters viz., Luminance, Luminance coefficient, Luminance uniformity and Contrast ratio.
\end{abstract}

Keywords: Liquid crystal, luminance, polarizing optical microscope, differential scanning calorimeter, scanning electron microscopy, X-ray diffraction.

Received: 26 June 2021

\section{Introduction}

Owing to the supreme properties of liquid crystals (LCs) like physical tuning and molecular designing, supramolecular hydrogen bonded liquid crystals (HBLCs) became most important materials in current research. Intermolecular hydrogen bonding between non-mesogenic-mesogenic materials, mesogenic-mesogenic materials or non-mesogenicnon-mesogenic materials produces the self-assembling of molecules, which induces new novel materials with different shapes and structures [1-11]. Further, these materials induce the new phases and physical properties. Such developments made hydrogen bonded LC's as essential materials for many applications such as optical storage devices, display devices, optical switches etc. Different types of HBLCs have been used by many authors in their research to derive different forms of applications.

Basically, display devices are specified in terms of chromaticity, i.e., black and white points. Where chromaticity is related to color information and black and white points, this represents luminance values $[12,13]$. This type of specification includes the determination of display parameters obtained from the color information and luminance of the novel materials. There are several spectroscopic and experimental methods to measure the color information and luminance of the LCs [13-15]. In previous work, LC texture were utilized to calculate desired display parameters. Display parameters like luminance, luminance coefficient, luminance uniformity and contrast ratio have been estimated from the optical textures through MATLAB software [16-18] To compute the display parameters, LCs' textures are used as function of temperature.

\section{Materials and methods}

\subsection{Synthesis of gold nanoparticles}

The gold nanoparticles are synthesized from the citrate reduction process. The chemical ingredients for synthesis, the reducing agent trisodium citrate dehydrate $\left(\mathrm{Na}_{3} \mathrm{C}_{6} \mathrm{H}_{5} \mathrm{O}_{7} \cdot 2 \mathrm{H}_{2} \mathrm{O}\right) 99 \%$ and hydrogen tetrachloroaurate (III) trihydrate $\left(\mathrm{HAuCl}_{4} \cdot 3 \mathrm{H}_{2} \mathrm{O}\right) \mathrm{ACS}, 99.99 \%$ were purchased from Sigma Aldrich laboratories, USA and used as such. From Inductively Coupled Plasma Optical Spectrometry (ICPOS) report (ICAP6500 Thermo Fisher Scientific make), the amount of gold present in solution of $1 \mathrm{ml}(1000 \mu \mathrm{l})$ of citrate capped gold nanoparticles is $96.75 \mu \mathrm{g}$. The author has dispersed very small amounts of citrate capped gold nanoparticles which contain the gold nanoparticles of amounts $4.83 \mu \mathrm{g}$, respectively. 


\subsection{Synthesis of silver nanoparticles}

$150 \mathrm{mg}$ of silver nitrate $\left(\mathrm{AgNO}_{3}\right)$ is taken into $30 \mathrm{ml}$ of distilled water and is mixed to the solution of $225 \mathrm{mg}$ of tetraoctylammonium bromide (TOAB) in $24 \mathrm{ml}$ of toluene. This solution is stirred continuously for one hour, and then $0.189 \mathrm{ml}$ of dodecanethiol is added and so obtained homogeneous solution is further stirred for $30 \mathrm{minutes}$. In $24 \mathrm{ml}$ of distilled water, $398 \mathrm{mg}$ of sodium borohydride (NaBH4) is dissolved and mixed with above obtained homogeneous solution.

This solution is stirred at room temperature for approximately three hours. From the solution, the organic phase is separated from aqueous solution and is reduced to 2 to $3 \mathrm{ml}$ of solution by evaporating in a roto-evaporator under vacuum at room temperature. Next $50 \mathrm{ml}$ of ethanol was added and the solution was centrifuged at $5000 \mathrm{rpm}$ for one hour. The supernatant liquid, which is dodecane thiol protected silver nanoparticles, was dissolved in $1 \mathrm{ml}$ of dichloromethane to get precipitated. This was washed with ethanol for many times for complete removal of organic material. $60 \mathrm{mg}$ of thiol capped silver nanoparticles were obtained after this centrifugation process.

\subsection{Homogeneous dispersion of gold and silver nano nanoparticles in LC compounds}

LC compound 6O.CB was obtained from Sigma-Aldrich laboratories, USA and used without further purification. For homogeneous dispersion, gold and silver nanoparticles were dissolved in toluene and stirred in magnetic stirrer (Remi make) for 1 hour. Then, this solution was added to isotropic state of $100 \mathrm{mg}$ of 6O.CB and the mixture is stirred well about 3 hours. Then the complex was cooled to room temperature which afforded 6O.CB with homogeneous dispersion of gold and silver nanoparticles. After cooling, 6O.CB mixtures were subjected to examination of both their textures and phase transition temperatures using polarizing optical microscope (POM) (SDTECHS make) attached with hot stage and differential scanning calorimetry (DSC) (Perkin Elmer Diamond DSC) along with the enthalpy values. The presence of gold and silver nanoparticlesNanoparticles in 6O.CB is characterized by the experimental techniques Scanning Electron Microscope (SEM) and XRD.

\subsection{POM (Polarising Optical Microscopy)}

Due to change in local molecular order, the LC molecule is characterized into different LC phases with an increase in temperature. Determination and characterization of these mesophase will give very important information on the textures and pattern of the LCs. The thermal ranges of nematic phase are slightly changed due to the dispersion of gold and silver nanoparticles in LC compounds and the textures of the phase changes by the self-assembly of nanoparticles.

\subsection{DSC Thermo grams}

DSC gives complete and accurate information about thermal history of liquid crystal melting points at different rates of cooling and heating. In this instrument an accurately weighted sample which is placed in an aluminum pan crimped tightly closed with a sealing press and placed in the sample chamber of DSC. In this process the instructions are provided with each type of instrument for obtaining a good DSC scan.

Small values of enthalpy gives the mesophase transitions involved in pre- or post-transitional or second order transitions. While increasing the concentration of nanoparticles at the nematic phase transition, the clearing temperatures are reduced. ItThis is the best complementary tool with the optical polarizing microscope. The DSC curve for the same is shown in Figs. $1-3$.

\subsection{Scanning Electron Microscope (SEM) Analysis}

To estimate the morphology and chemical composition of nanoparticles, SEM acts as a wonderful tool with image magnification range from $10 \mathrm{X}$ to $300,000 \mathrm{X}$ and the resolution is almost down to few nanometers. In addition to the topographical information, the elements present in LC compounds as well as dispersed nanocomposites can be obtained from SEM analysis. In SEM, a very small amount of LC Nano-composite material sample is sufficient for characterization studies, and homogeneous dispersion of citrate capped gold nanoparticles in the respective LC compounds are studied from the different parts of the compounds which gives the similar Energy Dispersive Spectroscopy (EDS) data.

SEM detects the spatial variations in chemical compositions by generating high resolution images of the shape of the metal nanoparticles. The chemical composition of the elements in terms of the atomic percent and weight in the given LC Nano-composites and SEM images of LC compounds are well studied from SEM analysis. The SEM and EDS of 6O.CB with the dispersed gold nanoparticles is shown in Fig. 4.The SEM and EDS data of 6O.CB with the dispersed silver nanoparticles is shown in Fig. 5. Results from data of EDS reveal the presence of chemical compounds in sample. Carbon(C), sulfur (S), silver (Ag) and oxygen $(\mathrm{O})$ elements are present in the 6O.CB with dispersed silver nanoparticle sample. 


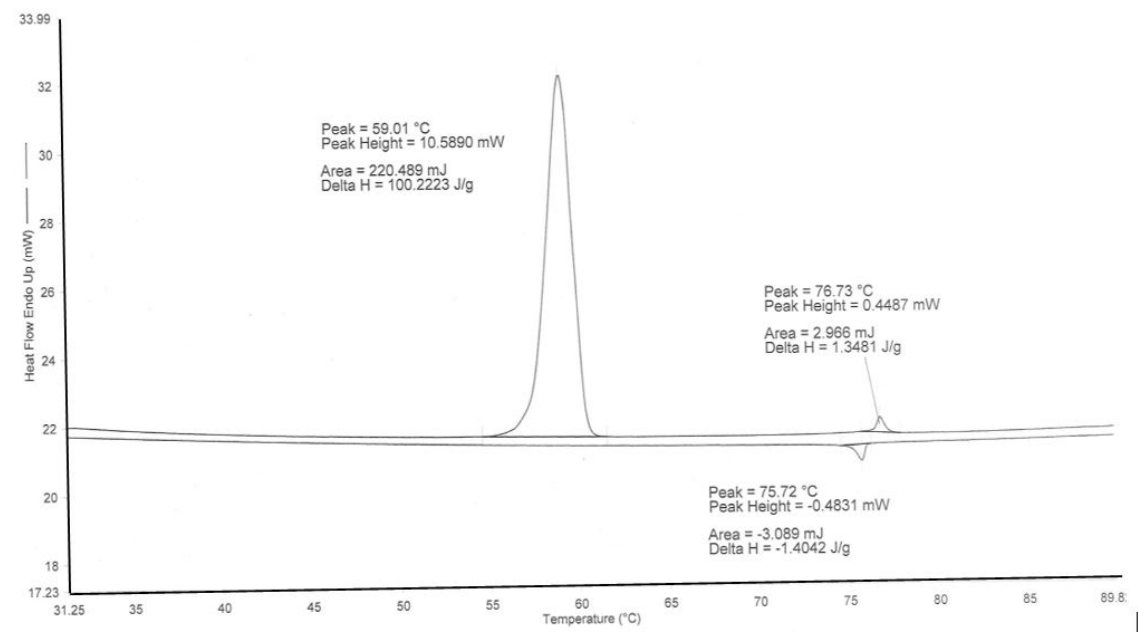

FIG. 1. DSC thermogram of 6O.CB pure

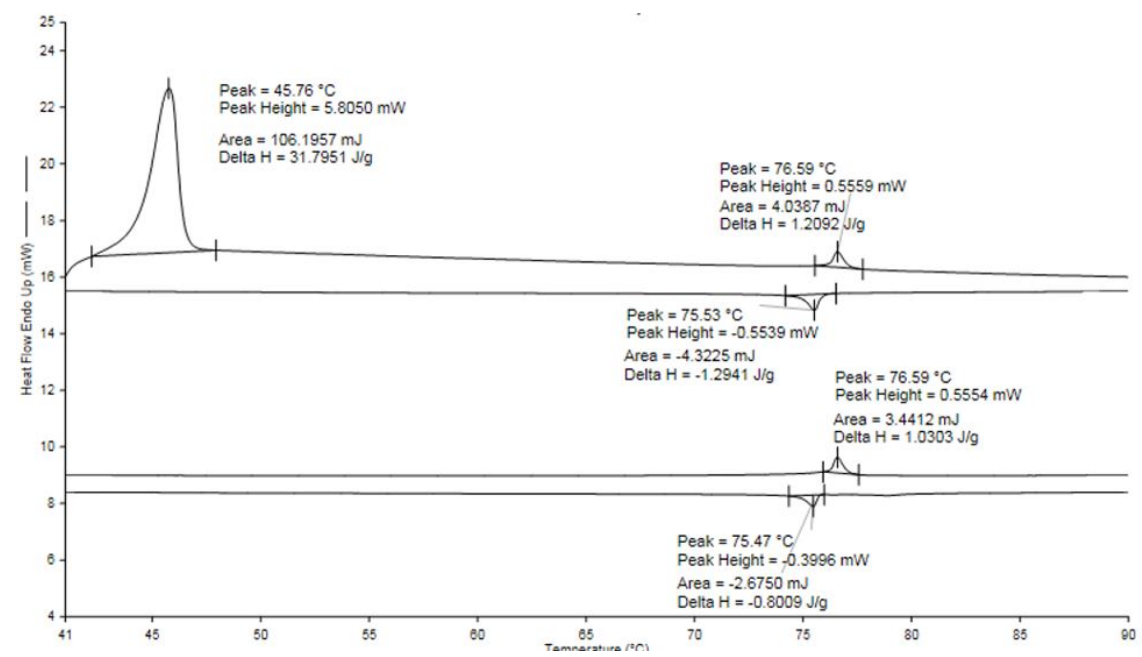

FIG. 2. DSC thermogram of 6O.CB with gold nanoparticles

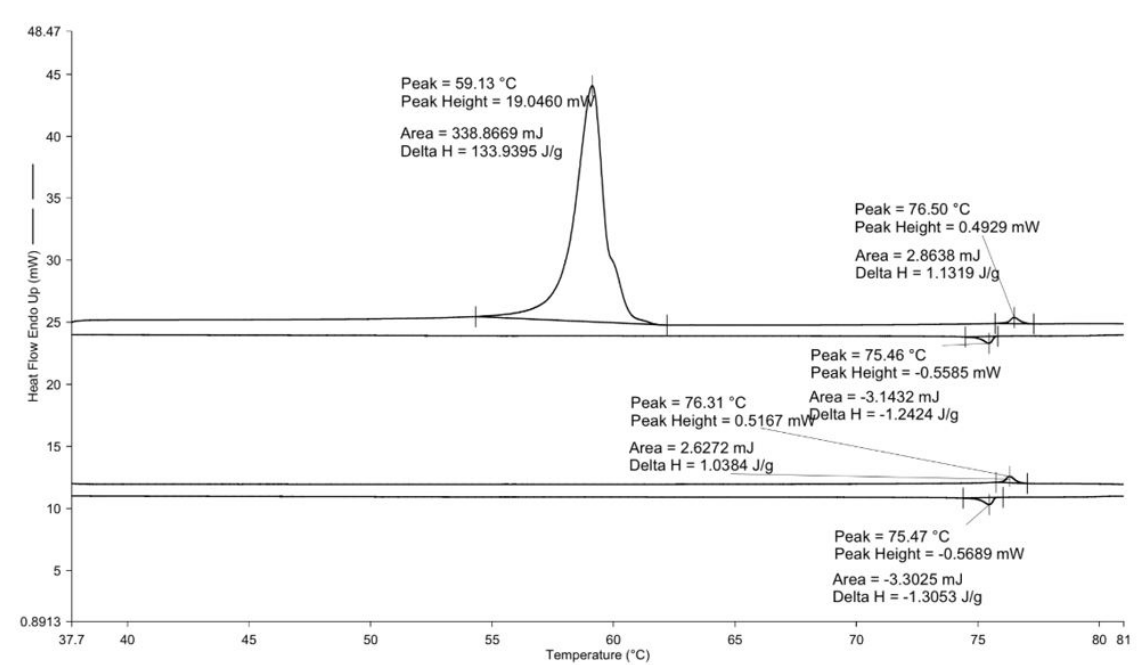

FIG. 3. DSC thermogram of 6O.CB with silver nanoparticles 

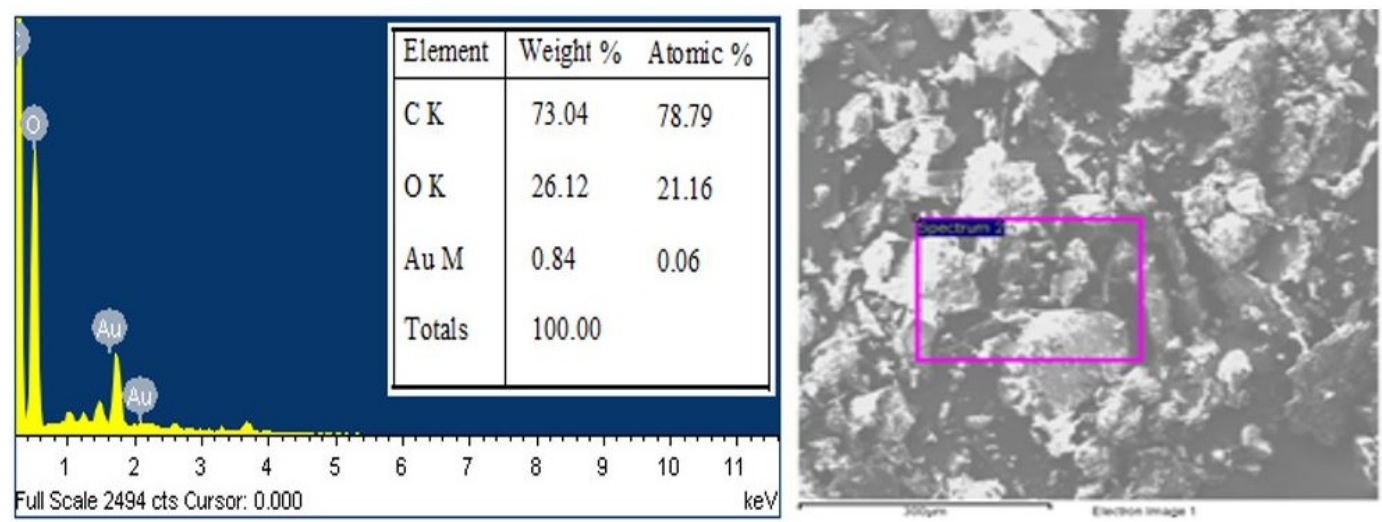

FIG. 4. SEM and EDS data of 6O.CB with the dispersed gold nanoparticles
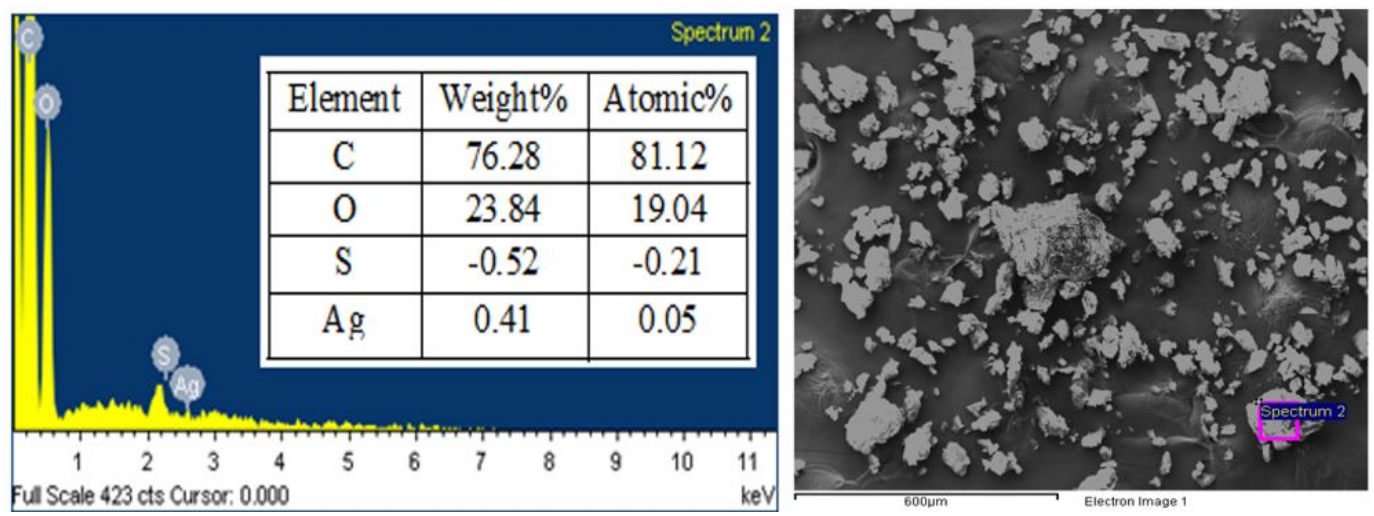

FIG. 5. SEM and EDS data of 6O.CB with the dispersed silver nanoparticles

\subsection{X-Ray diffraction (XRD) studies}

By utilizing XRD, the study of crystal structure of solids, defects, stresses, grain size and identity of the phases of LCs, by broadening of X-ray diffraction peaks. The Bragg's equation is given by:

$$
\lambda=2 d \sin \theta .
$$

Here, $d$ is the inter-planar spacing, $\lambda$ represents the wavelength of X-rays in which wavelength of the beam of $\mathrm{X}$-rays varying from 0.07 to $0.2 \mathrm{~nm}$ and $2 \theta$ is angle of diffraction.

A good amount of powder sample is required for X-ray diffraction to give the collective information about the size of the nanoparticles dispersed in LC materials. An ideal powder sample will provide a similar diffraction pattern in the isotropic, nematic and smectic phases.

When there are no inhomogeneous strains in the LC sample, the size of the crystal $t$, can be measured from Sherrer's formula,

$$
t=\frac{K \lambda}{\beta \cos \theta} .
$$

$K$ is Scherrer's constant and its value approaches to unity for a spherical crystal and 0.9 for LCs, $\lambda$ is wavelength of $\mathrm{X}$-rays, $\beta$ is the full width half maxima (FWHM) height of a diffraction peak and $\theta$ is diffraction angle.

The XRD data is taken for the 6O.CB with homogeneous dispersion of the gold nanoparticles is shown in Fig. 6. In comparison of JCPDS data peaks were well resolved and are matched with JCPDS card no. 03-0652870 which is clearly evidenced the existence citrate capped Au nanoparticles in LC compound 6O.CB. By using Scherrer's Formula, $t=\frac{k \lambda}{\beta \cos \theta}$, grain size $33 \mathrm{~nm}, \lambda=1.54 \AA, \beta=\mathrm{FWHM}$, Peaks at $38.39^{\circ}, 44.24^{\circ}$ and $64.55^{\circ}$ resembles the existence of citrate capped Au nanoparticles. The XRD data for 6O.CB with dispersion of silver nanoparticles is shown in Fig. 7. 


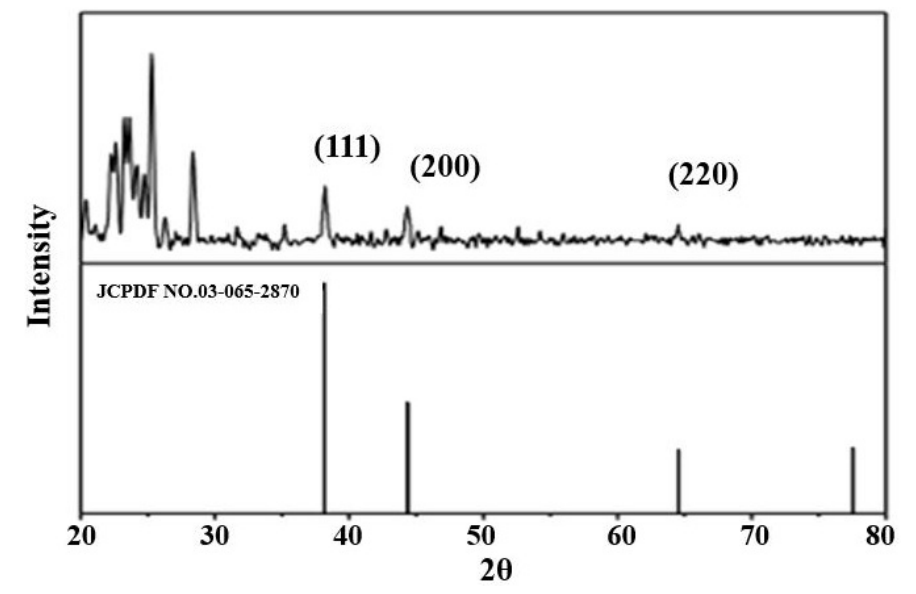

FIG. 6. XRD data of 6ocb pure and with the dispersion of gold nanoparticles
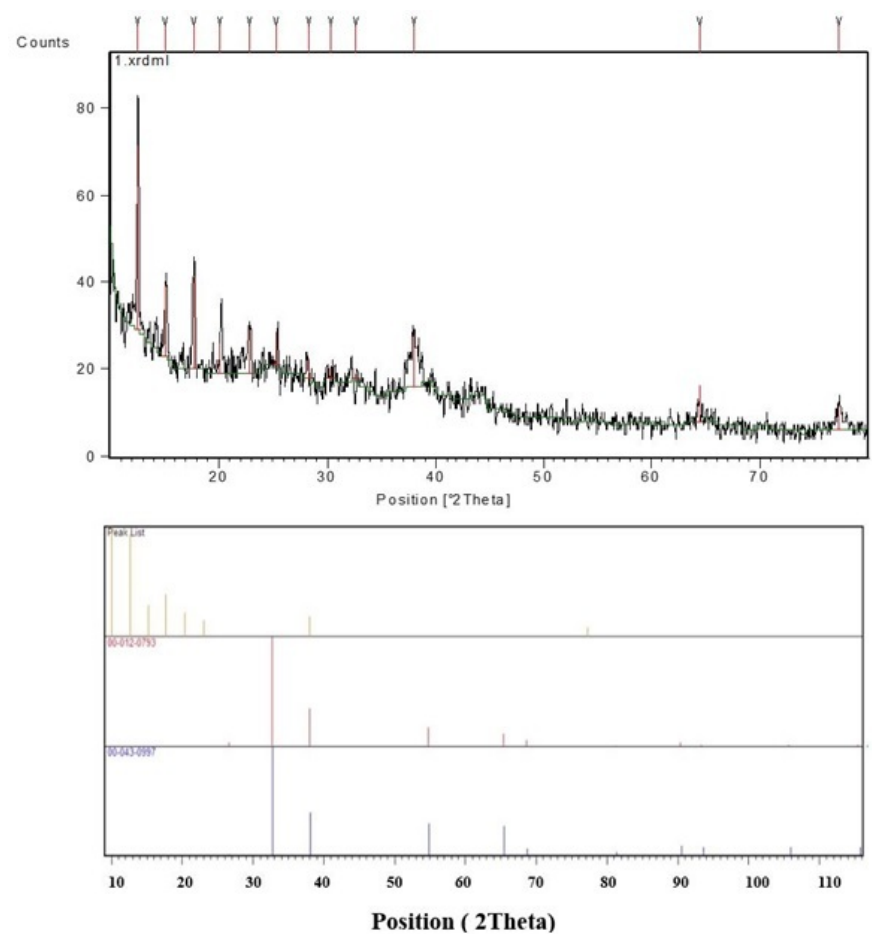

FIG. 7. XRD data of 6O.CB pure and with the dispersion of gold nanoparticles

\section{Theory}

The display device parameters of the LC compound images are calculated using MATLAB software [19]. The test image $I(m, n)$ is of the size $i \times j$ is recorded from POM with $i$ number of pixels in vertical direction and $j$ number of pixels in horizontal direction, where $m$ and $n$ are the spatial coordinates of the image. The total number of pixels can be computed as $N=i \cdot j$, where $0 \leq m \leq i, 0 \leq n \leq j$. The parameters for display device applications can thus be computed.

\subsection{Luminance}

The amount of light which is being transmitted or reflected from the object is being illuminated is called as luminance. In analysis of image, the weighted sum of intensities of color components $(R, G, B)$ is referred as luminance and is defined as [20,21]:

$$
L=0.2126 \cdot R+0.152 \cdot G+0.0722 \cdot B,
$$


where $L$ is luminance and $R, G, B$ are the color components of the image.

\subsection{Luminance coefficient}

The ratio of luminance to the illuminance of the image is called as luminance coefficient. Where illuminance of the image is referred as intensity of the incident light [23]. It can be computed from the components of parallel polarisation where no sample is there in POM:

$$
L_{C}=\frac{L}{\left(I_{L}\right)},
$$

where $L_{C}$ is luminance coefficient and $I_{L}$ is the illumination of the image.

\subsection{Luminance uniformity}

The percentage of luminance carried out from the one corner to another corner (pixel to pixel) of an image. The higher value of luminance uniformity provides the better consistency in brightness of the total image and which can be calculated as [22]:

$$
\Delta L_{U}=\left[\left(L_{\max }-L_{\min }\right)-1\right] \cdot 100,
$$

where $L_{U}$ is luminance uniformity.

\subsection{Contrast ratio}

The basic property of the display devices is contrast ratio. It is represented as the ratio between maximum to the minimum luminance of the recorded image from POM. Contrast in terms of luminance is defined as:

$$
C_{R}=\frac{\left(L_{\max }-L_{\min }\right)}{L_{\max }},
$$

where $C_{R}$ is contrast ratio and $L_{\max }$ and $L_{\min }$ are maximum and minimum luminance.

\section{Results and discussions}

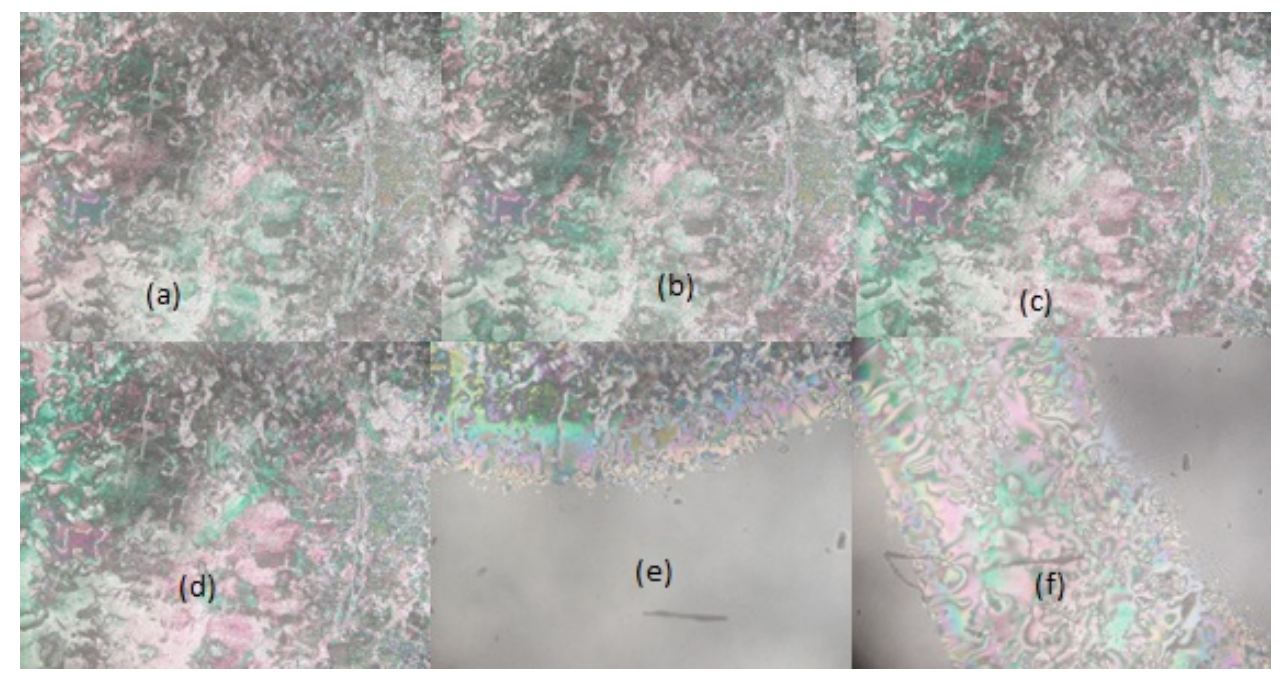

FIG. 8. 6O.CB pure Nematic phase textures at temperatures (a) $55{ }^{\circ} \mathrm{C}$, (b) $60{ }^{\circ} \mathrm{C}$, (c) $65{ }^{\circ} \mathrm{C}$, (d) $66^{\circ} \mathrm{C}$, (e) $74.4^{\circ} \mathrm{C}$ and (f) $74.7^{\circ} \mathrm{C}$

The nematic phase textures of the pure 6O.CB and dispersed with gold, silver nanoparticles are shown in Figs. $8-10$.

From the Figs. $11-14$ it is evident that the Luminance, Luminance coefficient, Luminance uniformity, and contrast ratio values are high at nematic phase for gold and silver nanoparticles as compared with pure 6O.CB. It is found that the percentage of increase in Luminance, Luminance coefficient, Luminance uniformity and contrast ratio for 6O.CB dispersed with gold nanoparticles are $8.57 \%, 9.813 \%, 31.52 \%$ and $27.96 \%$. Correspondingly for 6O.CB dispersed with silver Nanoparticles are $14.89 \%, 27.93 \%, 83.29 \%$ and $39.85 \%$. The higher luminance uniformity value offers well consistency in brightness of total image.

In our present studies silver nanoparticles dispersed in 6O.CB compound has higher value hence this may be a better material for display applications. Compare to gold silver nanoparticles dispersed in 6O.CB illustrates greater 


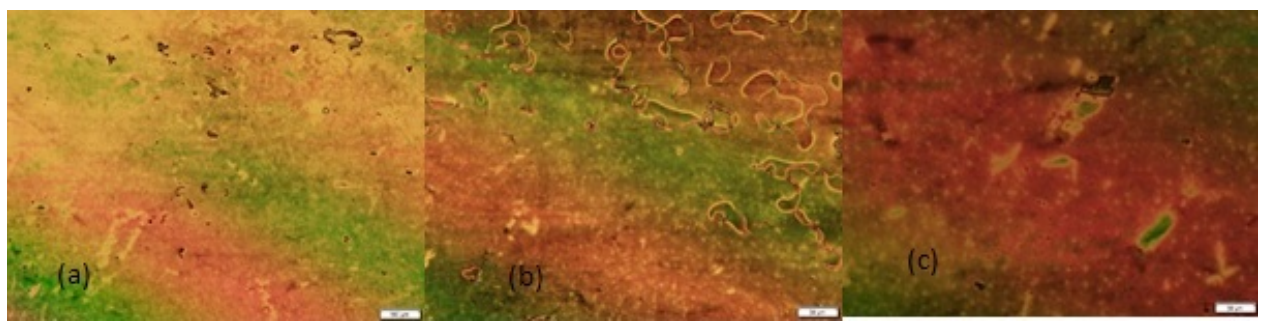

FIG. 9. 6O.CB with gold nanoparticles nematic phase textures at temperatures (a) $59.9{ }^{\circ} \mathrm{C}$, (b) $67{ }^{\circ} \mathrm{C}$ and (c) $73.4{ }^{\circ} \mathrm{C}$

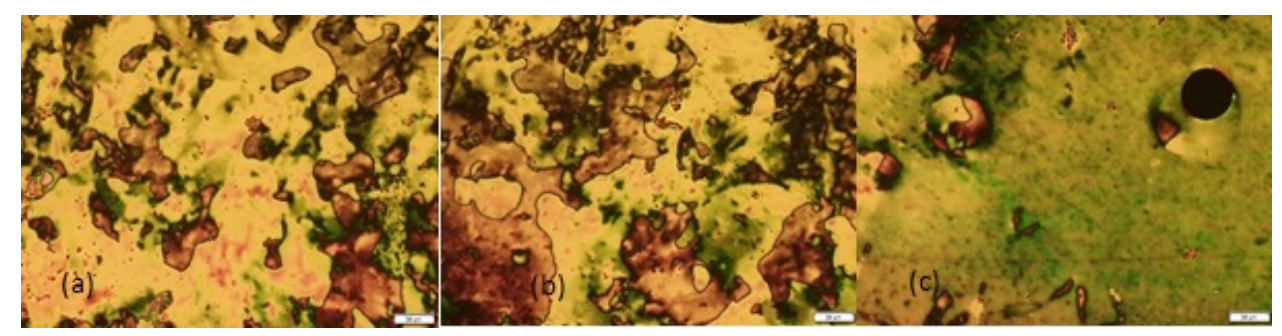

FIG. 10. 6O.CB with silver nanoparticles nematic phase textures at temperatures (a) $54.1{ }^{\circ} \mathrm{C}$, (b) $56.5^{\circ} \mathrm{C}$ and (c) $63.1^{\circ} \mathrm{C}$

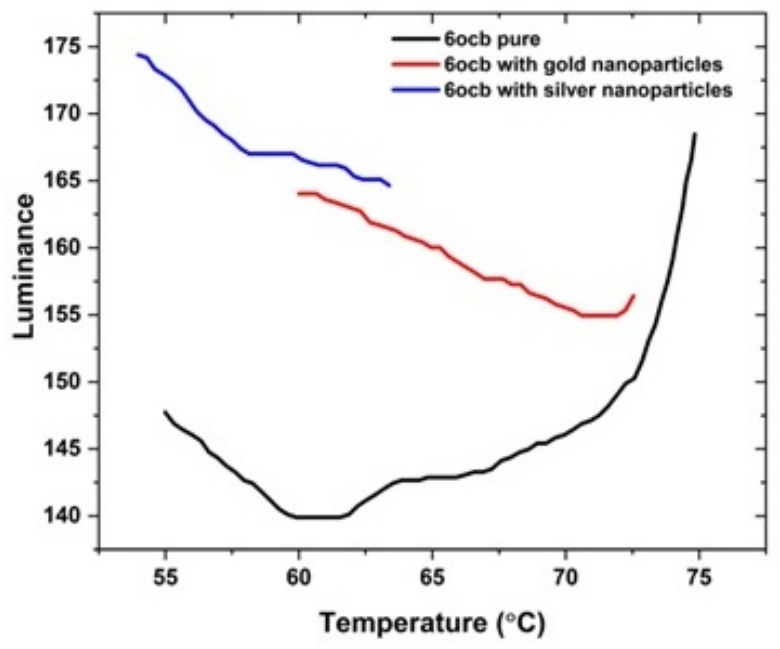

FIG. 11. Luminance values of the 6O.CB Pure and 6O.CB dispersed with gold, silver nano particles

values of above display parameters. This may be due to the fact that in visible region the reflection coefficient of silver is more than gold. For design of display devices luminance values play an important role. Higher the luminance the better is the compound for display device applications.

In alkoxy cyanobiphenyl liquid crystal compounds, good parallel molecular alignment exists in molecular structure due to the presence of oxygen atoms. Whereas in alkyl cyanobiphenyls in nematic phase there is imperfection in molecular arrangement [24]. Aligned molecules have the optical properties of uniaxial crystals this makes them exceptionally useful materials. Further the occurrence of oxygen atoms in molecular moiety increases the transition temperature.

By spectroscopic investigations, it is manifest that gold and silver nanoparticles are homogeneously dispersed in host 6O.CB liquid crystal compound. No variation in molecular structure and no substantial change in nematic phase due to the presence of gold and silver nanoparticles in mass material. 


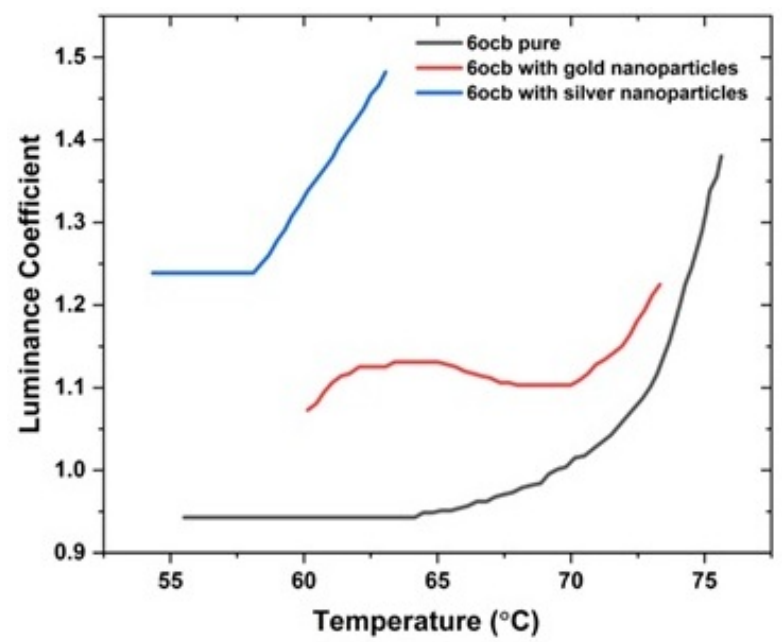

FIG. 12. Luminance uniformity values of the 6O.CB Pure and 6O.CB dispersed with gold, silver nano particles

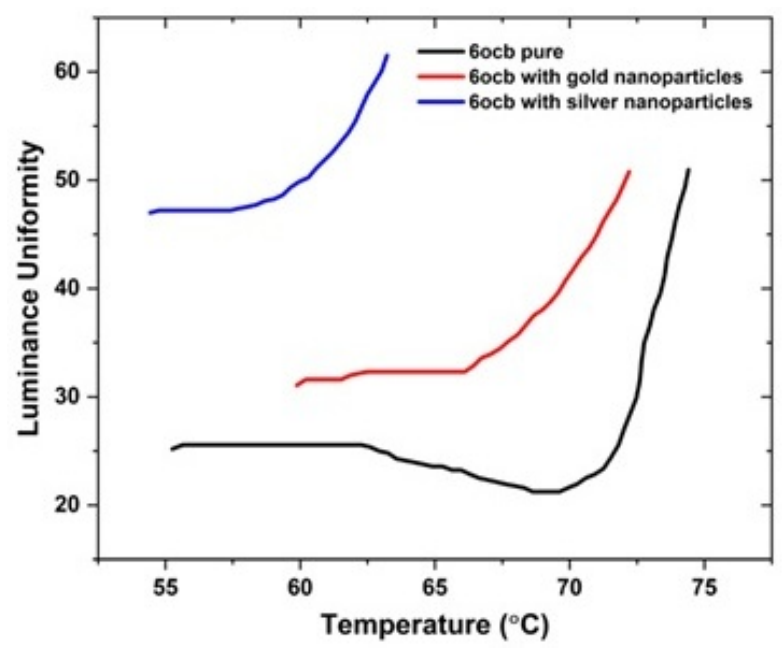

FIG. 13. Luminance Coefficient values of the 6O.CB Pure and 6O.CB dispersed with gold, silver nano particles

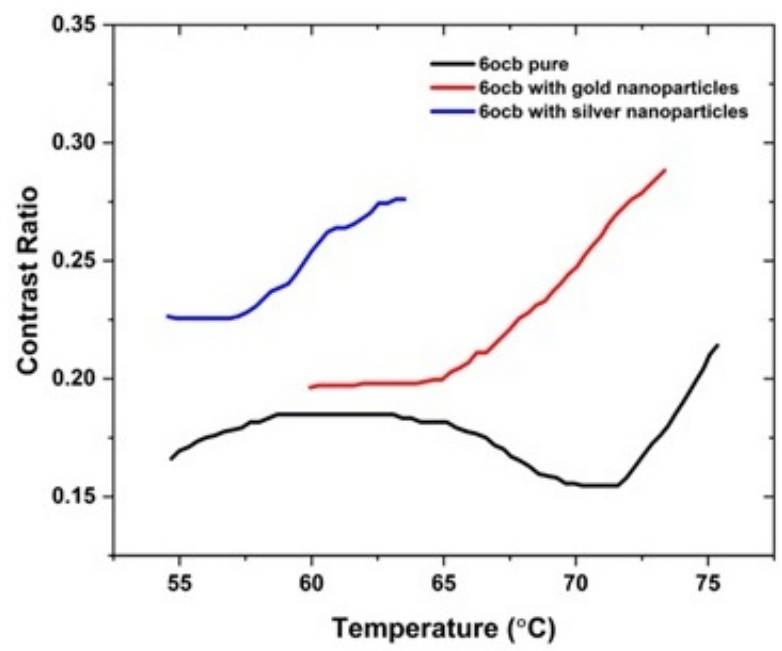

FIG. 14. Contrast Ratio values of the 6O.CB Pure and 6O.CB dispersed with gold, silver nanoparticles 


\section{Conclusion}

Display device parameters as a function of temperature for 6O.CB Pure and 6O.CB dispersed with gold, silver nnanoparticles are studied for LCD (Liquid Crystal Display) applications. The nematic transition temperatures are reduced by small amounts due to nano-dispersion. The conformation of gold and silver nanoparticles is evident by various spectroscopic techniques like SEM, and XRD studies. XRD studies confirm no alteration of structure of LCs by the dispersion of gold and silver nanoparticles. From the obtained parameters of luminance, luminance uniformity, luminance coefficient and contrast ratio, it is evident that the luminance values for the textures with dispersion of gold, silver nanoparticles have higher values than pure LC textures. It is concluded that nano-dispersed compounds are more suitable for display device applications as compared with pure LC.

\section{References}

[1] Prasad M.M., Mohan M.M.L.N., Chalapathi P.V., Ashok Kumar A.V.N., Potukuchi D.M. Influence of spacer and flexible chain on polymorphismin complementary hydrogen bonded liquid crystaldimers, SA: nOBAs. J Mol Liq., 2015, 207, P. $294-308$.

[2] Sangameswari G., Prabu N.P.S., Mohan M. A detailed study of hydrogen bonded ferroelectric mesogens formed between alkyl and alkyloxy benzoic acids with carbanyl glutamic acid. Liq Cryst., 2018, 45, P. 431-449.

[3] Ahmed H.A., Hagar M., Alaasar M., Naoum M. Wide nematicphases induced by hydrogen-bonding. Liq Cryst., 2019, 46(4), P. 550-559.

[4] Martinez-Felipe A., Cook A.G., Wallage M.J., Imrie C.T. Hydrogen bonding and liquid crystallinity of low molar mass and polymeric mesogens containing benzoic acids: a variable temperature Fourier transform infrared spectroscopic study. Phase Transitions, 2014, 87(12), P. 11911210 .

[5] Walker R., Pociecha D., Abberley J.P., Martinez-Felipe A., Paterson D.A., Forsyth E., Lawrence G.B., Henderson P.A., Storey J.M.D., Gorecka E., Imrie C.T. Spontaneous chirality through mixing achiral components: a twist-bend nematic phase driven by hydrogen-bonding between unlike components. Chem Comm., 2018, 54, P. 3383-3386.

[6] Goodby J.W., Pikin S.A., Robert Blinc,Clark N.A., Lagerwall S.T., Osipov M.A., Tadayoshi Sakurai, Yoshino K., Zeks B. Ferro electric liquid crystal-principles properties and applications. Gorden and Breech Science, Philadelphia, 1991.

[7] Percec V. Bioinspired supramolecular liquid crystals. Philos Trans R Soc A., 2006, 364, P. $2709-2719$.

[8] Yang D.K., Wu S.T. Liquid crystal materials in fundamentals of liquid crystal devices. JohnWiley and Sons, Ltd., Chichester(UK), 2006.

[9] Sreehari Sastry S., Nageswara Rao C., Vishwam. T., Mallika K., Ha sie Tiong. Confirmational Studies of intermolecular hydrogen bonding through induced crystal G phase innBA:7HB. Liq Cryst., 2013, 40(7), P. 932-941.

[10] Martinez-Felipe A., Imrie C.T. The role of hydrogen bonding in the phase behaviour of supra molecular liquid crystal dimers. J. Mol Struct., 2015, 1100, P. 429-437.

[11] Paterson D.A., Martinez-Felipe A., Jansze S.M., Antonius T.M.M., Storey J.M.D., Imrie C.T. New insights into the liquid crystal behaviour of hydrogen-bonded mixtures provided by temperature-dependent FTIR spectroscopy. Liq Cryst., 2015, 42, P. 928-939.

[12] Rohehrig H. Displays for medical imaging, Chapter 2: Physical characterization and image quality, dissertation. Arizona, The University of Arizona, 2018.

[13] Castellano J.A. Handbook of display technology. Academic Press Inc, San Diego(US), 1992.

[14] Vimal T., Dharmendra P.S., Agrahari K., Srivatsava A., Manohar R. Analysis ofoptical properties and mechanism of photoluminescence enhancement of a quantum dot - ferroelectric liquid crystal composite. Photonics Lett Poland, 2016, 8, P. $23-25$.

[15] Pathaka G., Agraharia K., Roya A., Srivatsavaa A., Strzezyszb O., Garbatb K., Manohara R. Dispersion of fluorescent dye in the nematic liquid crystal: enhanced photoluminescence and high birefringence. Opto-electron rev., 2018, 26, P. 317-324.

[16] Chen J., Cranton W., Fihn M. Handbook of Visual Display Technology. Springer International Publishing, Switzerland, 2016.

[17] Widdel H., Post D.L. Color in electronic displays. Plenum press, New York, 1992.

[18] Sweet Khisa. Characterization and calibration of a LCD display based on a limited set of color samples (master Thesis). Saint-Étienne, University of Jean Monnet, 2010.

[19] Gonzalez R.C., Woods R.E., Eddins S.L. Digital image processing using MATLAB. Knoxville(TN), Gatesmark Publishing, 2009.

[20] toplightco.com (internet). London (UK), lighting blog (cited 2015 June 18). Available from: https://toplightco.com/blog/whats-the-differencebetweenluminance-and-illuminance.html

[21] cs.stackexchange.com (internet). New York (US), Stackover flow (cited 2018 May 31 ). Available from: https://cs.stackexchange.com/questions/92569/what-is-the-difference-between-luma-and luminance.html

[22] displayalliance.com (internet). Amesbury (Essex), Mass Integrated, Inc (cited 2011 July 8). Available from: http://www.displayalliance.com/glossary/luminanceuniformity.html

[23] Casol M., Fiorentin P., Scroccaro A.16-th IMEKO TC4Symposium- Exploring New Frontiers of Instrumentation and Methods for Electrical and Electronic Measurements. Florence, Italy, 2008, Sept 22-24.

[24] Ghanadzade A., Beevers M.S. Effect of surface and molecular structure on the dielectric behaviour in thick layers of cyanobiphenyl liquid crystals. Journal of Molecular Liquids, 2002, 100(1), P. 47-57. 